\title{
Encadeamentos da Doença Renal Crônica e o impacto na qualidade de vida de pacientes em hemodiálise
}

\author{
Chains of Chronic Kidney Disease and the impact on quality of life of hemodialysis patients \\ Cadenas de enfermedad renal crónica y el impacto en la calidad de vida de los pacientes en hemodi- \\ álisis \\ Wanderson Alves Ribeiro" ${ }^{*}$, Denilson da Silva Evangelista ${ }^{2}$, Júlio César Figueiredo Júnior ${ }^{3}$, Julio \\ Gabriel Mendonça de Sousa ${ }^{4}$
}

Como citar esse artigo. Ribeiro, WA; Evangelista, DS; Júnior, JCF; De Sousa, JGM. Encadeamentos da Doença Renal Crônica e o impacto na qualidade de vida de pacientes em hemodiálise. Revista Pró-UniverSUS. 2020 Jul./Dez.; 11 (2): $111-120$

\begin{abstract}
Resumo
A doença renal crônica (DRC) trata-se de enfermidade caracterizada pela perda permanente e irreversível das funções dos rins, comumente associada a diabetes mellitus e hipertensão arterial e que pode progredir para uma fase mais severa, chamada de doença renal crônica terminal (DRCT). Trata-se de uma pesquisa bibliográfica de abordagem qualitativa e caráter descritivo. Como metodologia, utilizou-se a Biblioteca Virtual de Saúde, nas bases de informações LILACS, BDENF, MEDLINE e SCIELO, com recorte temporal de 2011 à 2018. O estudo objetivou em descrever os encadeamentos da Doença Renal Crônica na qualidade de vida de pacientes em hemodiálise e caracterizar o impacto na qualidade de vida de pacientes em hemodiálise. Após a leitura reflexiva dos artigos encontrados, emergiram 4 categorias: Doença Renal Crônica; As complexidades da Hemodiálise (HD) e da Fístula arteriovenosa (FAV); O impacto na Qualidade de vida dos pacientes com Doença Renal Crônica; O enfermeiro e sua atuação frente ao quadro clínico do paciente em uso de hemodiálise. Por fim, conclui-se que a complexidade no atendimento a pacientes com DRC se dá, em especial, pela abordagem do tratamento que será adotada. Isso se dá pelo fato de que ao se diagnosticar a Doença renal crônica (DRC), será preciso avaliar também a viabilidade dos tratamentos disponíveis, existência de outras doenças, além dos fatores sociais e emocionais que possam ter influência sobre a terapêutica.
\end{abstract}

Palavras-chave: Hemodiálisem Enfermagem, Qualidade de Vida.

\begin{abstract}
Chronic kidney disease (CKD) is a disease characterized by permanent and irreversible loss of kidney function, commonly associated with diabetes mellitus and high blood pressure, which may progress to a more severe phase, called terminal chronic kidney disease (CKD). . This is a bibliographical research with qualitative approach and descriptive character. As methodology, we used the Virtual Health Library, in the databases LILACS, BDENF, MEDLINE and SCIELO, with timeframe from 2011 to 2018. The study aimed to describe the links of Chronic Kidney Disease in the quality of life of patients in hemodialysis and characterize the impact on quality of life of hemodialysis patients. After reflective reading of the articles found, four categories emerged: Chronic Kidney Disease; The complexities of Hemodialysis (HD) and Arteriovenous Fistula (AVF); The impact on quality of life of patients with chronic kidney disease; The nurse and his role in the clinical situation of patients on hemodialysis. Finally, it is concluded that the complexity of care for patients with CKD is due in particular to the treatment approach that will be adopted. This is due to the fact that when diagnosing chronic kidney disease (CKD), it will also be necessary to evaluate the feasibility of available treatments, the existence of other diseases, as well as the social and emotional factors that may influence the therapy.
\end{abstract}

Keywords: Hemodialysis, Nursing, Quality of Life.

\section{Resumen}

La enfermedad renal crónica (ERC) es una enfermedad caracterizada por la pérdida permanente e irreversible de las funciones renales, comúnmente asociada con diabetes mellitus e hipertensión arterial y que puede progresar a una fase más grave, llamada enfermedad renal en etapa terminal (ERC). Esta es una investigación bibliográfica con un enfoque cualitativo y carácter descriptivo. Como metodología, se utilizó la Biblioteca Virtual de Salud, en las bases de información de LILACS, BDENF, MEDLINE y SCIELO, con un marco de tiempo de 2011 a 2018. El objetivo de este estudio fue describir las cadenas de la enfermedad renal crónica en la calidad de vida de los pacientes en hemodiálisis y caracterizan el impacto en la calidad de vida de los pacientes en hemodiálisis. Después de la lectura reflexiva de los artículos encontrados, surgieron 4 categorías: enfermedad renal crónica; Las complejidades de la hemodiálisis (HD) y la fístula arteriovenosa (AVF); El impacto en la calidad de vida de los pacientes con enfermedad renal crónica; La enfermera y su desempeño en vista del estado clínico del paciente en hemodiálisis. Finalmente, se concluye que la complejidad en el cuidado de pacientes con ERC ocurre, en particular, debido al enfoque de tratamiento que se adoptará. Esto se debe al hecho de que al diagnosticar la enfermedad renal crónica (ERC), también será necesario evaluar la viabilidad de los tratamientos disponibles, la existencia de otras enfermedades, además de los factores sociales y emocionales que pueden influir en la terapia.

Palabras clave: Hemodiálisis, Enfermería, Calidad de Vida.

Afiliação dos autores:

${ }^{1}$ Enfermeiro. Docente do Curso de Graduação da UNIG e UCB. Mestre pelo Programa Acadêmico em Ciências do Cuidado em Saúde pela Escola de Enfermagem Aurora de Afonso Costa da UFF, Niterói/RJ. Brasil. E-mail: nursing_war@hotmail.com ORCID: https://orcid.org/0000-0001-8655-3789

${ }^{2}$ Enfermeiro. Universidade Iguaçu. Preceptor do curso de graduação em Enfermagem da UNIG. Brasil. E-mail: denilsonevan@gmail.com ORCID: https://orcid.org/0000-0002-72820361

${ }^{3}$ Enfermeiro. Faculdade de Saúde Ibituruna - FASI. Brasil. E-mail: julio.enf_@hotmail.com ORCID: https://orcid.org/0000-0003-3406-1234

${ }^{4}$ Acadêmico do curso de graduação em Enfermagem da Escola de Enfermagem Anna Nery/UFRJ. Brasil. E-mail: juliogabriel33@gmail.com ORCID: https://orcid.org/0000-00028013-3369 


\section{Introdução}

A doença renal crônica (DRC) trata-se de enfermidade caracterizada pela perda permanente e irreversível das funções dos rins, comumente associada a diabetes mellitus e hipertensão arterial e que pode progredir para uma fase mais severa, chamada de doença renal crônica terminal (DRCT). Nesse estágio, as funções renais são nulas, de modo que se torna necessário o tratamento por meio da hemodiálise (HD), a fim de se substituir as funções dos rins ${ }^{1}$.

Os rins são os principais órgãos responsáveis pela regulação da homeostase, mantendo constante o volume hídrico, a composição química e o pH sanguíneo, como também é responsável pela manutenção da pressão arterial. Tendo como unidade funcional os néfrons que possui uma região especifica denominada glomérulo, no qual é responsável pela filtração do plasma sanguíneo ${ }^{2}$.

Cabe ressaltar que os rins são um par de órgãos, castanho-avermelhados, localizados no plano retroperitoneal, sobre a parede posterior do abdômen, desde a $12^{\mathrm{a}}$ vértebra torácica à $3^{\mathrm{a}}$ vértebra lombar no adulto, tem $12 \mathrm{~cm}$ de comprimento, $6 \mathrm{~cm}$ de largura e 2,5 de espessura ${ }^{3}$.

Cada rim contém aproximadamente um milhão de néfrons, que são suas unidades funcionais. Os néfrons consistem em um glomérulo contendo as arteríolas aferentes e eferentes, cápsula de Bowman, túbulo contorcido proximal, alça de Henle, túbulo contorcido distal, e dutos coletores ${ }^{4}$.

Cerca de $80 \%$ do sangue não é diretamente filtrado, chegando a arteríola eferente direcionando-se para a circulação peritubular. A composição do filtrado assemelha-se ao plasma sanguíneo, no entanto com uma quantidade mínima de componentes como proteínas e macromoléculas, evidenciando-se que devido ao seu tamanho há uma dificuldade no processo de filtração glomerular ${ }^{2}$.

Posteriormente o plasma filtrado segue através dos túbulos renais no qual haverá ainda mais uma alteração no volume, e composição resultante de processos como a reabsorção tubular que ocorre ao longo dos túbulos renais, alça de Henle e túbulo distal, tendo como finalidade a recuperação de substancias essencial ao organismo, que foram filtradas do plasma sanguíneo ${ }^{5}$.

A função básica dos rins é limpar o plasma sanguíneo de substâncias indesejáveis ao organismo, como as proteínas finais do metabolismo, ureia, creatinina, ácido úrico e uratos, através da filtração ${ }^{4}$.

Entre outras funções estão: regulação do equilíbrio hidroeletrolítico e do equilíbrio ácido-básico, regulação da pressão arterial, produção de eritropoetina (regulação da produção de eritrócitos), síntese de vitamina D e secreção de prostaglandinas 5 .

Em que a urina é formada nos néfrons através de um complexo processo de três etapas: filtração glomerular, reabsorção tubular e secreção tubular, sendo que os dois últimos envolvem, frequentemente, o transporte passivo e ativo. Contudo a glicose aparece na urina (glicosúria) quando a quantidade de glicose e o filtrado glomerular excedem a quantidade que os túbulos são capazes de reabsorver ${ }^{6}$.

O rim tem função de filtrar as toxinas do corpo e excretá-lo pela urina. Como consequência do declínio da função renal, o rim apresenta dificuldade de regulação e 16 excreção dos produtos finais do metabolismo, fazendo com que os mesmos fiquem acumulados na corrente sanguínea, levando ao quadro conhecido como uremia, que gradativamente afeta todos os sistemas do organismo, logo, quanto menor a taxa de filtração glomerular dessas toxinas, maior é a percepção dos sinais e sintomas ${ }^{4}$.

Dessa forma, entende-se que a DRC é uma enfermidade de nível global, uma vez que não interfere apenas na fisiologia do paciente, mas em seu cotidiano também, na forma como ele lida com a realidade ao seu redor, sendo impossibilidade de realizar as mesmas atividades de antes com alguma normalidade. A DRC acaba por desencadear uma série de sinais e sintomas em todo o organismo, podendo favorecer limitações e cronicidade da doença em seu tratamento, tendo como resultado limitações físicas, sociais e emocionais, que interferem de modo significativo na qualidade de vida dos portadores de $\mathrm{DRC}^{1}$.

Dados do censo 2016 sobre diálise no Brasil, disponibilizados pela Sociedade Brasileira de Nefrologia (SBN), estimavam que, naquele ano, mais de 122 mil pacientes estavam em tratamento dialítico no país um aumento de cerca de três vezes a quantidade de pacientes no mesmo tipo de tratamento, em 2000. Entre as causas primárias que conduzem o paciente à diálise, destacam-se a idade (mais comum em idosos), obesidade, diabetes, hipertensão arterial ou pacientes com histórico familiar de DRC. Esses compõem os principais grupos de riscos para DRC, necessitando, assim de maior atenção por parte dos profissionais da saúde e dos órgãos responsáveis pela implementação de políticas públicas visando a atenção à pacientes dialíticos ${ }^{7}$.

Estudos esclarecem que os tratamentos disponíveis nas doenças renais terminais são: hemodiálise, diálise peritoneal e transplante renal e estas modalidades são complexas, variadas e representam um problema de saúde de ampla magnitude e relevância, especialmente quando se reconhece sua complexidade, seus riscos, diversidade de opções e custo ${ }^{8}$.

Nesse sentido, que dizer que a hemodiálise é o método de diálise mais comumente empregado para remover substâncias nitrogenadas tóxicas do sangue e excesso de água. Requer cuidado intensivo devido à possibilidade de intercorrências clínicas". As autoras 
ressaltam a importância de se levar em consideração o papel do enfermeiro no atendimento a pacientes crônicos, em especial, no que diz respeito à qualidade da assistência prestada e na educação em saúde 9 .

A detecção precoce e o tratamento adequado em estágios iniciais ajudam a prevenir os desfechos deletérios e a subsequente morbidade relacionados às nefropatias. Ademais, resultam em potenciais benefícios para qualidade de vida, longevidade e redução de custos associados ao cuidado em saúde ${ }^{10}$.

Importante destacar que a falta de informação e a ausência de sintomas nos estágios iniciais da doença agravam o quadro da patologia, considerando que suas manifestações só ocorrem quando o rim já perdeu $50 \%$ de sua função, o que leva a maioria dos casos a um diagnóstico tardio. Na prática, isso significa que o paciente necessitará de diálise ou mesmo um transplante renal, o que poderia ser evitado tendo-se o conhecimento prévio da doença e suas complicações ${ }^{11}$.

Dentre os profissionais de saúde envolvidos no tratamento do paciente o enfermeiro tem seu papel importante, pois é este profissional que estará participando ativamente de todos os processos com este cliente, que poderá ter sua qualidade de vida alterada, devido à fatores como: Ansiedade, depressão, dificuldade de lidar com a doença irreversível e incurável dando a este suporte para melhor qualidade de vida ${ }^{12}$.

O paciente renal crônico se depara com diversos conflitos que causam modificações no seu cotidiano com restrições e comprometimento da sua qualidade de vida $^{13}$.

Diante da problemática exposta, surge a seguinte questão norteadora: Qual o impacto da Doença Renal Crônica na qualidade de vida dos paciente em hemodiálise?

Para tal, o artigo tem como objetivo descrever os encadeamentos da Doença Renal Crônica na qualidade de vida de pacientes em hemodiálise e caracterizar o impacto na qualidade de vida de pacientes em hemodiálise.

\section{Metodologia}

Trata-se de uma pesquisa bibliográfica de abordagem qualitativa e caráter descritivo. Cabe ressaltar que a pesquisa bibliográfica que é desenvolvida com auxílio de material já elaborado, constituído principalmente de livros e artigos científicos. Contudo em grande parte dos estudos seja exigido algum tipo de trabalho deste gênero, há pesquisas desenvolvidas exclusivamente a partir de fontes bibliográficas ${ }^{14}$.

Os dados foram coletados em base de dados virtuais. Para tal utilizou-se a Biblioteca Virtual de Saúde(BVS), na seguinte base de informação: Literatura Latino-Americana e do Caribe em Ciências da Saúde
(LILACS), Literatura Internacional em Ciência da Saúde (MEDLINE), Scientific Eletronic Library Online (SCIELO), dentre outros, no período de junho à agosto de 2019.

Optou-sepelosseguintesdescritores:Hemodiálise; Enfermagem; Qualidade de Vida que se encontram nos Descritores em Ciência da Saúde (DECS).

Estabeleceu-se então para a realização da pesquisa os critérios de inclusão: textos na íntegra e em português com abordagem da temática estabelecida e que obedecessem ao recorte temporal de 2011 a 2018 e como critérios de exclusão, os textos incompletos e em língua estrangeira, textos que não abordassem a temática estabelecida e com recorte temporal inferior a 2011.

Após a associação de todos os descritores foram encontrados 322 artigos e selecionados apenas 18 artigos.

Figura 1. Fluxograma das referências selecionadas

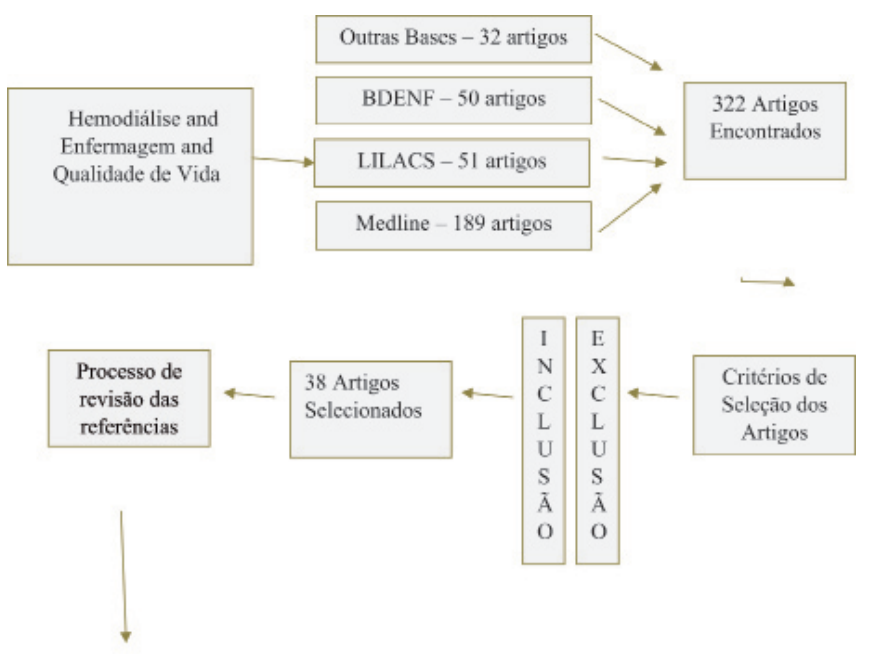

18 Artigos Selecionados

Fonte: Produção dos autores.

\section{Análise de Dados e Discussão de Resultados}

Subsequente a esta seleção, foi realizado uma leitura reflexiva dos artigos, onde descreveram-se os resultados encontrados nesta leitura e, ainda, uma discussão sucinta relacionada aos achados.

Posterior à leitura reflexiva dos ensaios supracitados e o agrupamento dos resultados encontrados emergiram quatro categorias: Doença Renal Crônica; As complexidades da Hemodiálise (HD) e da Fístula arteriovenosa (FAV); O impacto na Qualidade de vida dos pacientes com Doença Renal Crônica; O enfermeiro e sua atuação frente ao quadro clínico do paciente em uso de hemodiálise. 


\section{Doença Renal Crônica}

A DRC, atualmente, tornou-se um problema de saúde pública segundo dados epidemiológicos que apontam que a doença alcançou delineamentos espantosos. No Brasil, determina-se que cerca de 1,4 milhões de indivíduos possuem algum grau de perda de função renal. Estudos populacionais em países distintos apresentam prevalência de DRC de 7,2\% para indivíduos acima de 30 anos e $28 \%$ a $46 \%$ em indivíduos acima de 64 anos. Em estágios avançados, a DRC está associada ao crescimento de internações hospitalares, grande índice de mortalidade por doenças cardiovasculares, elevado impacto na comodidade de vida e alto custo para a saúde pública ${ }^{7}$.

Os rins têm como principais funções a manutenção da estabilidade homeostática relacionado a eletrólitos, líquidos e solutos orgânicos, em consequência da variação na excreção urinária de água e íons, como o sódio, cloreto, potássio, fosfato, cálcio e magnésio. Os rins também expelem produtos metabólicos finais, como uréia, fosfatos, ácido úrico, sulfatos, substâncias como drogas e medicamentos, além de serem responsáveis pela secreção e produção hormonal e enzimática, como a eritropoetina, a renina e o calcitrio $1^{15}$.

A Doença renal ocorre quando os rins não conseguem remover os resíduos metabólicos do corpo ou realizar as funções reguladoras. As substâncias que normalmente são eliminadas na urina acabam se acumulando nos líquidos corporais em consequência de excreção renal prejudicada, levando a uma ruptura nas funções metabólicas e endócrinas, bem como distúrbios hídricos, eletrolíticos e ácidos básicos. A Doença renal pode ser aguda ou crônica e é uma doença que tem repercussão sistêmica e é uma via final comum de muitas doenças do trato urinário ${ }^{16}$.

A Doença Renal Crônica (DRC) define-se como a perda progressiva e geralmente irreversível da função renal de depuração, ou seja, da filtração glomerular. Caracteriza-se pela deterioração das funções bioquímicas e fisiológicas de todos os sistemas do organismo, secundária ao acúmulo de catabólitos (toxinas urêmicas), alterações do equilíbrio hidroeletrolítico e ácido básico, acidose metabólica, hipovolemia, hipercalemia, hiperfosfatemia, anemia e distúrbio hormonal, hiperparatireoidismo, infertilidade, retardo no crescimento, entre outros ${ }^{17}$.

A perda gradativa do ritmo de filtração glomerular (RFG) constatada na DRC resulta na redução das funções regulatórias, excretórias e endócrinas comprometendo o funcionamento de todos os órgãos do corpo humano ${ }^{18}$.

Vale ressaltar que, a DRC é caracterizada pela deterioração da função renal, com consequente retenção de substâncias nitrogenadas no sangue, desequilíbrio resultante da concentração inadequada de solutos, acúmulo de substâncias tóxicas não excretadas pela urina e deficiência na síntese de hormônios específicos ${ }^{19}$.

Em consonância ao contexto, a DRC é uma doença que tem várias consequências na vida do paciente e de difícil terapêutica, pois traz com ela sérias implicações físicas, psicológicas e socioeconômicas, onde não só atinge o indivíduo, mas também a família e a comunidade. Além disso, essa patologia é uma enfermidade que modifica todo o cotidiano do paciente que a vivência, sendo também caracterizada como um grave problema social, onde interfere nas funções que esse indivíduo desempenha na sociedade ${ }^{20}$.

Vale mencionar que, a Doença renal crônica é uma patologia que afeta os diferentes aspectos da vida do paciente. É de difícil tratamento, com sérias implicações físicas, psicológicas e socioeconômicas não apenas para o indivíduo, como também para a família e a sociedade ${ }^{21}$.

Sendo a DRC uma condição clínica caracterizada pele retenção de toxinas urêmicas, associada à perda irreversível da função renal. É sabido que as toxinas urêmicas afetam várias partes do corpo, incluindo o cérebro e, consequentemente, ocasionando uma neuropatia periférica e disfunção no Sistema Nervoso Central $^{22}$

A DRC é uma doença silenciosa nos estágios inicias. As pessoas que apresentam algum grau de lesão renal em estágios iniciais pode não apresentar sinais ou sintomas que alerta a doença, assim retardando o diagnóstico, não tendo assim a eficácia nos tratamentos e o prognóstico.Com essas observações que se fica evidente que o uso de testes laboratoriais são de tamanha importância para o diagnóstico, os quais, devem ser de baixo custo e disponibilizados na maioria dos laboratórios de análises clínicas ${ }^{23}$.

Para o tratamento dos pacientes portadores da doença renal é preciso o entendimento dos fatores diversos, que se relacionam englobando a doença de base, o estágio da doença, a velocidade da diminuição da filtração glomerular (FG), identificação de complicações e comorbidades, principalmente as cardiovasculares ${ }^{11}$.

As técnicas renais substitutivas também chamadas de rins artificiais têm sido utilizadas por quase 40 anos para tratar pacientes portadores de Doença renal grave. Em certos tipos de Doença renal aguda, a diálise é utilizada simplesmente para manter o paciente por algumas semanas até haver resolução de lesão renal. $\mathrm{Na}$ atualidade, as técnicas de diálise foram desenvolvidas a tal ponto que milhares de pessoas com Doença renal permanente vêm sendo mantidas com saúde por vários anos ${ }^{5}$.

Por sua vez, a diálise peritoneal tem como objetivo remover as substância tóxicas, resíduos metabólicos e restabelecer o equilíbrio hidroeletrolítico normal. A diálise peritoneal pode ser realizada de diferentes formas: diálise peritoneal intermitente aguda ou crônica; diálise peritoneal ambulatorial contínua (CAPD); diálise 
peritoneal cíclica contínua $(\mathrm{CCPD})^{16}$.

Corroborando ao contexto, hemodiálise é um processo de limpeza dos produtos de degradação acumulados do sangue. É realizado em pacientes que sofrem com Doença renal em estágio terminal ou nos pacientes de maior gravidade que necessitam de diálise a curto prazo 6 .

A cirurgia renal pode incluir nefrectomia parcial ou total, transplante renal para a doença renal em estágio terminal (DRET). A nefrectomia constitui também o procedimento de escolha para remover um rim saudável para doação a um receptor de transplante. A ausência de um rim não resulta em comprometimento da função renal quando o rim remanescente é normal ${ }^{16}$.

\section{As complexidades da Hemodiálise (HD)}

Dentre as formas de tratamento da doença renal crônica está a Hemodiálise (HD). Segundo a Sociedade Brasileira de Nefrologia esta é a modalidade de tratamento realizado através da ligação do usuário a um dialisador, que substitui a função renal, promovendo a filtração das toxinas sanguíneas e eliminando o excesso de líquido corpóreo ${ }^{7}$.

A hemodiálise é o tratamento terapêutico mais utilizados na DRC e é acompanhado de inúmeras restrições de vida tais como, restrições alimentares, de atividades diárias básicas, e sociais. A forma ativa do tratamento é realizada em hospitais ou unidades especializadas, onde o usuário necessita dispor de cerca de quatro horas por dia, durante três vezes por semana, o que implica diretamente na sua qualidade de vida ${ }^{24}$.

De acordo com a Sociedade Brasileira de Nefrologia, cerca de $30 \%$ da população em tratamento hemodialítico é composta por pessoas idosas. A HD em pessoas idosas apresenta alguns problemas particulares, devido a presença de maior número de comorbidades, fragilidade e a dificuldade do estabelecimento do acesso vascular, condição necessária para a ligação do usuário ao dialisador ${ }^{24,25}$.

A circulação extracorpórea da HD consiste em um sistema de difusão e osmose, em que o usuário se liga a uma máquina que bombeia o sangue corpóreo, até o dialisador que age como uma membrana substituindo os glomérulos e os túbulos renais, retendo as toxinas, o excesso de eletrólitos e efetuando o balanço hidroeletrolítico, e ao final devolve o sangue para o usuário. Esse processo é repetido por inúmeras vezes, filtrando o sangue ao máximo possível ${ }^{4}$.

Esse tratamento demanda normalmente da disponibilidade de três a quatro vezes por semana durante 3 a 4 horas por dia, em local apropriado, geralmente em clínica especializada ou hospitais ${ }^{25}$.

É um processo complexo e especializado que necessita da adequação de materiais e equipamentos, capacitação dos profissionais de saúde e preparo do paciente. O tratamento depende da presença de acesso vascular eficiente. Na hemodiálise, o sangue é desviado do paciente para uma máquina onde, através de um dialisador, ocorre a filtração extracorpórea. A remoção do excesso de líquidos e a depuração do sangue com a retirada de ureia, fósforo, ácido úrico e moléculas médias, ocorre através dos processos de difusão, ultrafiltração e convecção ${ }^{27}$.

A transferência de solutos ocorre entre o sangue e a solução de diálise através de uma membrana semipermeável artificial por três mecanismos: a difusão, que é o fluxo de soluto de acordo com o gradiente de concentração, sendo transferida massa de um local de maior concentração para um de menor concentração, isso depende do peso molecular e características da membrana. A ultrafiltração é a remoção de líquidos através de um gradiente de pressão hidrostática e a convecção é a perda de solutos durante a ultrafiltração, quando ocorre o arraste de solutos na mesma direção do fluxo de líquidos através da membrana ${ }^{27}$.

A hemodiálise evita a morte dos pacientes com Doença renal crônica, embora ela não cure a doença renal e não compense a perda das atividades endócrinas ou metabólicas dos rins. Os pacientes que recebem hemodiálise devem submeter-se ao tratamento pelo resto da vida (usualmente três vezes por semana, por um mínimo de três a quatro horas por tratamento), ou até se submeterem a um transplante renal.

Os acessos são classificados como acessos vasculares temporários, permanentes e fístula arteriovenosa. $\mathrm{O}$ acesso vascular nos pacientes com Doença renal crônica pode ser temporário ou permanente. $\mathrm{O}$ acesso venoso temporário pode ser usado para uma diálise única, utilizado por poucas horas, ou por meses, onde é utilizado enquanto se aguarda uma fístula arteriovenosa ficar madura. E o acesso permanente, provê o fluxo adequado para a prescrição de diálise, dura um longo período, e tem um baixo índice de complicações ${ }^{28}$.

A Fístula arteriovenosa (FAV) Desenvolvido em 1966 por Appel, esse método era utilizado para casos agudos e limitados a poucas sessões de hemodiálise, dada a dificuldade imposta pelas repetidas punções e o rápido esgotamento dos acessos. O uso de (cateteres, como os cateteres de longa permanência) para o acesso vascular, está relacionado $18 \mathrm{com}$ aumento da mortalidade em até $50 \%$, quando comparado ao uso das fístulas em pacientes renais crônicos. Além disso, podem deteriorar o sistema venoso dos pacientes impedindo a confecção das fístulas ${ }^{29}$.

A Fístula Artério Venosa é o método permanente de acesso vascular, para o portador de DRT e que realizam continuamente este tratamento. Para sua formulação 
que consiste na união de uma artéria (radial) com uma veia (cubital mediana), normalmente no antebraço sendo necessário um procedimento cirúrgico. Essa fístula é puncionada toda vez que o usuário necessita do tratamento, onde sua funcionalidade arterial serve para a retirada do sangue para o dialisador e o venoso serve para infusão do sangue dialisado. Para utilização dessa via é necessário a espera de dois a três meses para sua maturação, ou seja, para que ocorra a dilatação venosa devido à pressão exercida pelo sangue vindo da artéria ${ }^{4}$.

Durante as sessões de hemodiálise, duas agulhas são inseridas na fístula arteriovenosa, uma que remove o sangue do corpo do paciente para o dialisador e a outra que devolve o sangue filtrado para o corpo do paciente. Para se ter sucesso no tratamento de hemodiálise é necessário se ter um adequado acesso vascular, uma técnica muito utilizada é a Rope-Ladder, que alterna os sítios de punção, mas existe uma nova técnica que vem se popularizando pelo mundo, chamada técnica de Buttonhole, iniciada há quase 30 anos na Europa e no Japão, atualmente é amplamente aprovada e usada no Pacífico Noroeste e, com base em comunicações com equipes de atendimento de pacientes de todo o país e do Canadá ${ }^{30}$.

\section{O impacto na Qualidade de vida dos pacientes com Doença Renal Crônica}

Vale ressaltar que, a qualidade de vida (QV) vem se alterando ao passar dos anos. No princípio, foi utilizado para expor o sucesso de bens materiais, acrescentando para estimação do desenvolvimento econômico da sociedade, para que sejam estabelecidos vários indicadores econômicos e elaborados instrumentos para que a qualidade de vida seja avaliada, mensurada ${ }^{32}$.

Fala, em QV, como a duração ou extensão da vida, existindo alguns que associam-na a um estilo de vida de um determinado grupo, com nível econômico mais elevado, podendo também estar associada à satisfação com atributos físicos e emocionais, bem como relacionada com o conceito de bem-estar pessoal e social, e ainda a aspectos econômicos ${ }^{32}$.

Assim a QV está relacionada com uma avaliação global das condições de vida dos indivíduos, dependendo da benevolência da vida, ao mesmo tempo um balanço entre a posição social dos indivíduos.

Contudo, a qualidade de vida relacionada à saúde dos pacientes renais crônicos em terapias renais substitutivas (hemodiálise e diálise peritoneal) apresentou-se globalmente diminuída, principalmente em relação aos aspectos físicos, em todas as faixas etárias; enquanto que domínios como saúde mental, aspectos sociais e emocionais foram preservados ${ }^{3,13,4}$.
Corroborando ao contexto, o termo qualidade de vida está relacionado ao impacto do estado de saúde sobre a capacidade do indivíduo de viver plenamente, entretanto estão incluídas nesta definição uma variedade potencial de condições que afetam sua percepção, seus sentimentos e comportamentos relacionados ao seu funcionamento diário, assim como à sua condição de saúde e intervenções médicas5,3.

A DRC traz consigo mudanças inimagináveis no cotidiano dos pacientes, havendo necessidade de mudança seu hábito alimentar. Tendo estes pacientes que evitar a ingesta de sal, gorduras, excesso de líquido, exigindo um balanceamento e um controle rigoroso antes não vivenciado pelo mesmo. Essa mudança junto com as restrições é elaborada para que esse paciente tenha qualidade de vida. Para que isso ocorra é necessário o apoio à colaboração e às instruções sejam realizadas de maneira clara e objetiva onde o profissional de saúde irá realizar a ação educativa desse paciente e de sua família ${ }^{3,6}$

Corrobora-se que essa modificação ocorrerá não somente em sua alimentação, ocorrerá também no seu trabalho, em sua atividade física e em seu lazer. Esse bloqueio em suas atividades passa a atingir dimensões na vida social desse indivíduo fazendo com que o seu prazer e a sua alegria de viajar, estar com sua família seja interrompida ${ }^{23}$.

Assim a importância do apoio familiar parece decisiva para uma avaliação positiva dos pacientes sobre a sua qualidade de vida, pois além do apoio familiar a sensação de bem estar oriunda do tratamento auxilia no tratamento das restrições e dos pensamentos negativos ${ }^{12}$.

$\mathrm{O}$ fornecimento adequado de alimentação favorece a manutenção, a prevenção e a recuperação do estado nutricional evitando futuras complicações metabólicas a este paciente, o enfermeiro deverá ter o olhar clínico para possíveis cronicidades da doença, implicações, limitações, criando planos terapêuticos e estratégicos para o enfrentamento tendo seu papel ativo no tratamento do paciente renal crônico. Esse acompanhamento enfermeiro/paciente se dá de forma relutante até a chegada esperada de sua autonomia, reconhecimento pessoal e estabilidade psicológica de modo que fique evidenciado suas habilidades, competências e domínio de si mesmo ${ }^{3,7}$.

Nesse contexto, o termo Qualidade de Vida compreende uma ampla gama de conceitos que afetam a satisfação global com a vida, como boa saúde, moradia adequada, emprego, segurança, educação e lazer. Quando relacionada à saúde, leva em conta os aspectos físicos, sociais e emocionais causadas por uma doença ou tratamento.

A qualidade de vida relacionada à saúde dos pacientes renais crônicos em terapias renais substitutivas (hemodiálise e diálise peritoneal) apresenta-se 
globalmente diminuída, principalmente em relação aos aspectos físicos, em todas as faixas etárias; enquanto que domínios como saúde mental, aspectos sociais e emocionais foram preservados ${ }^{2,13}$.

O impacto do tratamento dialítico na qualidade de vida é um critério importante para avaliar esta e outras intervenções na área da saúde, além de analisar o impacto das doenças crônicas no cotidiano das pessoas. Os avanços tecnológicos e terapêuticos na área de diálise foram inicialmente voltados para a avaliação da sobrevida e sinais de doença renal crônica ${ }^{38}$.

Entre os demais problemas que ocorrem no paciente com Doença Renal que dificultam o tratamento e pioram a qualidade de vida estão: diminuição do interesse sexual, medo da morte, restrições dietéticas e hídricas, alteração da imagem corporal e cuidados específicos com a higiene, tornando necessário o fornecimento de suporte social e educacional como parte integrante do tratamento. Desordens do sono são comuns em pacientes com DRC, sendo as mais mencionadas: insônia, síndrome da apneia do sono, síndrome das pernas inquietas, problemas esses que afetam a quantidade, a latência e duração do sono ${ }^{3,9}$.

A doença faz com que o paciente sinta diversas emoções para posteriormente se estruturar e dar sentido à sua vida; o processo de alteração cognitiva, funcional e psicológica, que caracterizam o pesar também trazem repercussões para qualidade de vida do paciente renal crônico e é representado por estágios (negação, raiva, barganha, depressão e aceitação) que são enfrentados de maneira particular por cada paciente ${ }^{4,10}$.

Ao se deparar frente à patologia existente, $\mathrm{o}$ paciente entra em conflito interno gerando sentimentos que ameaça sua autoimagem gerando estresse e conflito que poderão interferir de maneira negativa na adesão de sua terapia. O paciente em tratamento hemolítico está completamente dependente dos profissionais devidamente capacitados e da tecnologia que é o uso das máquinas representando ali a manutenção de sua homeostase.

Mesmo com todos os processos envolvidos na hemodiálise e suas complexidades é necessário que a equipe de enfermagem além de executar procedimentos e técnicas, utilizem a empatia por esse paciente atuando também na perspectiva de um cuidado humanizado. Estabelecer relacionamentos interpessoais faz parte do cuidado humanizado, favorecendo com isso, condições agradáveis e favoráveis a esse tratamento. Esse paciente poderá encontrar-se emocionalmente fragilizado e a equipe multidisciplinar quando bem treinados $\mathrm{e}$ capacitados poderá aliviar medos e pensamentos negativos.

O paciente encontra a dificuldade no tratamento tendo como fatores principais: limitações do lazer, a aceitação da doença, a interferência profissional no trabalho, a restrição hídrica, a dificuldade de entendimento da doença bem como o horário e tempo de duração da diálise ${ }^{4,1}$.

Referente à compreensão dos pacientes no processo de hemodiálise, estudos relatam a falta de entendimento sobre a doença tendo o percentual de 76,6\% dos pacientes que apresentam baixa escolaridade, o que comprova que a falta de conhecimento e informação interfere diretamente em seu tratamento ${ }^{4,2}$.

Esse processo torna-se desgastante, pois o paciente será submetido à realizar três dias por semana durante um período de quatro horas por dia, impossibilitando a prática de suas atividades antes exercidas.

Nesse momento segundo a relação afetiva e o apoio familiar é essencial uma vez que assume proteção, desenvolve-se um sistema de valores e crenças". Essa é a hora em que o profissional em saúde deve "realizar estratégias educativas encorajando a ter uma vida ativa com amigos e familiares dentro de suas limitações ${ }^{12}$.

Estudos concluíram que a DRC e os tratamentos não constituem impedimentos direto ao trabalho, mas causa limitações importantes ao paciente ocasionando no decorrer da doença afastamentos e até mesmo aposentadoria.

Estudos relatam que a atividade produtiva é algo essencial na vida do ser humano, principalmente naqueles que estão na sua fase de máxima capacidade de produzir ${ }^{4,7}$.

Com isso, o novo estilo de vida a ser adotado pela pessoa submetida à hemodiálise pode originar sentimentos como medo, ansiedade, insegurança, culpa e raiva. Como consequência, há a probabilidade de uma diminuição da autoestima e de um comportamento de resistência em seguir o tratamento adequadamente, prejudicando, assim, o quadro clínico ${ }^{4,4}$.

Para tal, torna-se essencial conhecer a percepção do paciente sobre sua situação, para que o profissional possa aprimorar a qualidade da assistência prestada, auxiliando-o no enfrentamento das limitações que o tratamento leva, promovendo a autonomia e encorajando o autocuidado ${ }^{4,7}$.

Embora promovam a manutenção e o prolongamento da vida, as terapias renais substitutivas, às quais os pacientes renais crônicos são submetidos, não oferecem uma cura para a doença e, em longo prazo, acabam por prejudicar a vida cotidiana e a QV do paciente.

Vale ressaltar que os pacientes em tratamento hemodialítico apresentam limitações no seu cotidiano e vivenciam inúmeras perdas e mudanças biopsicossociais, tais como perda da saúde (em nível fisiológico e bioquímico), anemia, perda de competência física, cognitiva e sexual, perda do emprego e outras atividades/funções, dependência de tratamento médico e da terapia renal, além de alterações físicas e na imagem corporal, restrições dietéticas e hídricas, associadas ao tratamento ${ }^{4,7}$. 
Corroborando ao contexto, a doença renal crônica (DRC), afecção de caráter progressivo e irreversível, acarreta uma série de desarranjos bioquímicos, clínicos e metabólicos, responsáveis direta ou indiretamente por altas taxas de hospitalização, morbidade e mortalidade. Juntamente ao diagnóstico e tratamento, com seus consequentes impactos fisiológicos e emocionais, diversas perdas acontecem nos âmbitos profissional, social, sexual e psicológico ${ }^{4,7}$.

Assim, conviver com a perda da liberdade e da capacidade produtiva para o trabalho e para suas atividades diárias, e o enfrentamento da mudança radical em seu aspecto físico é visto de modo negativo.

Tão logo, a apresentação de sinais e sintomas decorrentes da realização do tratamento e do esquema de hemodiálise (três vezes por semana por um período de quatro horas) impõe aos pacientes perdas significativas na saúde e no vigor físico.

Além da sintomatologia apresentada, os pacientes precisam conviver com as alterações físicas como a FAV (fístula artéria venosa) e o cateter de duplo-lúmen foram os que mais geraram repercussões negativas em suas vidas ${ }^{4,8}$.

Estudos apontaram que pacientes após receberem o diagnóstico da DRC, precisam de uso inicial do cateter duplo-lúmen logo após a confecção FAV para a efetivação do tratamento hemodialítico. Fator esse estressante e causador de vergonha ao despertar olhares curiosos

Vale mencionar que, compete ao profissional enfermeiro colaborar, estimular, a adesão ao tratamento estabelecido, formando assim, vínculo e permeando informações de modo que o paciente compreenda e tenha suas dúvidas esclarecidas, promover conforto e ajudar a adequação do seu novo estilo de vida amenizando seu nível de estresse existente, ajudando-lhe a cooperar com o tratamento proposto.

Corroborando ao contexto, o enfermeiro nefrologista deverá ser simpático, tolerante, solícito, sensível, mantendo a humildade e a compaixão, proporcionando ao DRC conforto necessário para aceitar o tratamento ${ }^{4,8}$.

\section{$O$ enfermeiro e sua atuação frente ao quadro clínico do paciente em uso de hemodiálise}

De acordo com Frazão (2014), os enfermeiros são responsáveis por tornarem o ambiente confortável e adequado para os cuidados pessoais, além de prepararem a sessão de hemodiálise cuidadosamente, gerenciando a máquina, mistura de fluídos e a monitorização dos sinais vitais, utilizando técnicas corretas de punção, cuidados com a máquina de hemodiálise e avaliar a taxa de filtração a fim de prevenir infecções ${ }^{13}$.
Gerenciar o tratamento desde a admissão até a alta, avaliar constantemente os exames laboratoriais, trocas de curativos e observar as manifestações corporais, promovendo, dessa forma, a segurança e a proteção do paciente em hemodiálise.

O vínculo enfermeiro-paciente família é extremamente relevante. Permitindo que a confiança e o respeito se estabeleçam. Ao conhecer a família do paciente adquire-se subsídios para orientá-lo, esclarecer suas dúvidas e amenizar sua ansiedade possibilitando troca de experiência, vínculo e conhecimento de maneira mútua, promovendo uma melhor adesão ao tratamento.

Para isso, torna-se necessário que o enfermeiro utilize meios de comunicação favoráveis e adequado fazendo-se compreender facilitando a aceitação ao tratamento prolongado, estabelecendo um vínculo terapêutico. Adquirindo com isso a confiabilidade e o alívio no processo de hemodiálise.

No tocante à tomada de decisão compartilhada torna-se instrumento de grande valor possibilitando a promoção e o autocuidado desse cliente.

O enfermeiro torna-se responsável pela orientação adequadasobreadoença, suascomplicaçõesdirecionando o paciente para o melhor plano terapêutico.

Uma das medidas em que o enfermeiro necessita estabelecer rigorosamente é avaliar se o paciente está seguindo corretamente a dieta estabelecida pesando antes e após a diálise, mantendo sempre os registros atualizados no prontuário do paciente para que se tenha uma comparação semanal de ganho de peso. Assim como também ter o controle sobre suas eliminações intestinais.

\section{Considerações Finais}

Conclui-se que a complexidade no atendimento a pacientes com DRC se dá, em especial, pela abordagem do tratamento que será adotada. Isso se dá pelo fato de que ao se diagnosticar a Doença renal crônica (DRC), será preciso avaliar também a viabilidade dos tratamentos disponíveis, existência de outras doenças, além dos fatores sociais e emocionais que possam ter influência sobre a terapêutica.

Este estudo fundamentou-se na ideia de que a informação é o melhor tratamento. Por este motivo, pensar no enfermeiro como agente educador do paciente hemolítico é um dos princípios que nortearam a elaboração deste trabalho, uma vez que é este profissional que, no dia-a-dia hospitalar, tem maior contato e relação com o paciente. Assim sendo, a justificativa deste trabalho se sustenta sobre o pilar da educação como via de melhoria do bem-estar físico e emocional do paciente hemolítico, através da capacitação dos enfermeiros como responsáveis pelo processo de educação do paciente acerca de sua condição clínica, tratamento e 
mudanças dos hábitos de vida.

É um constructo multidimensional, onde passa a englobar toda uma organização física, psicológica e social. Essa percepção de qualidade de vida não abrange diretamente com o status funcional objetivo de um paciente que passa muitas vezes a ser influenciado pelos níveis de ansiedade na qual seu suporte social é modificado pela adequação da diálise e das comorbidades físicas.

O paciente com DRC não tem ou tem pouco conhecimento de sua condição, o que, além de um problema por si só, pode levar ao diagnóstico tardio de seu estado, inviabilizando sua cura, estabelecendo a cronicidade de sua doença.

Por fim, dessa forma é fundamental a adaptação familiar ao tratamento desse paciente tendo compromisso e dedicação absorvendo todas as informações necessárias impertinentes ao caso clínico do cliente, salientando que essa complementação com o serviço de saúde resultará em uma reabilitação favorável a esse paciente.

\section{Referências}

1. Aires M. M. (2012) Fisiologia(*), 4ª ed., Ed. Guanabara Koogan/ GEN, Rio de Janeiro, RJ. ISBN: 9788527721004

2. Costa DN. Os benefícios da técnica de Buttonhole em pacientes renais crônicos. Recife. Monografia [Especialização em Enfermagem em Nefrologia] - Faculdade de Boa Viagem e CCE - Centro de Capacitação Educacional; 2015. 37f

3. Smeltzer SC. Bare C. Brunner \& Suddarth: et al. Tratado de Enfermagem Médico-Cirurgica. 12. ed. Rio de Janeiro: Guanabara Koogan, 2017. v.3.

4. Hall, J. E. (2011) Guyton \& Hall: Tratado de Fisiologia Médica (*), $12^{\mathrm{a}}$ ed., Ed. Elsevier, Rio de Janeiro, RJ. ISBN: 978-85-352-3735-1

5. Bastos MG, Bregman R, Kirsztajn GM. Doença renal crônica: frequente e grave, mas também prevenível e tratável. Rev. Assoc. Med. Bras. [Internet]. 2010 [citado 2020 Abr 25]; 56(2): 248-253. Disponível em: http://www.scielo. br/scielo.php?script=sci_arttext\&pid=S0104-42302010000200028\&lng=en. https://doi.org/10.1590/S0104-42302010000200028.

6. Silva AS, Silveira RS, Fernandes GFM, Lunardi VL, Backes VMS Percepcõos e mudanças na qualidade de vida de pacientes submetidos à hemodiálise. Rev. bras. enferm. [Internet]. 2011 Oct [citado 2020 Abr 27]; 64( 5 ): 839-844. Disponível em: http://www.scielo.br/scielo.php?script=sci arttext\&pid=S0034-71672011000500006\&lng=en. https://doi.org/10.1590/ S0034-71672011000500006.

7. Frazão CMFQ, Delgado MF, Araújo MGA, Silva FBBL, Sá JD, Lira ALBC. Cuidados de enfermagem ao paciente renal crônico em hemodiálise. Rev Rene. [Internet]. 2014 [citado 2020 Abr 25]15(4):701-709. 18. Disponível em: https://www.redalyc.org/pdf/3240/324032212018.pdf

8. Gil, A.C. Métodos e técnicas de pesquisa social. 6. ed. São Paulo. Ed. Atlas, 2008. ISBN 978-85-224-5142-5

9. Smeltzer SC. Bare C. Brunner \& Suddarth: et al. Tratado de Enfermagem Médico-Cirurgica. 12. ed. Rio de Janeiro: Guanabara Koogan, 2017. v.3.

10. Nepomuceno FCL, Melo JIM, Silva EA, Lucena KDT. Religiosidade e qualidade de vida de pacientes com insuficiência renal crônica em hemodiálise. Saúde debate [Internet]. 2014 Mar [citado 2020 Abr 27] $38(100$ ): 119-128. Disponível em: http://www.scielo.br/scielo. php?script=sci arttext\&pid=S0103-11042014000100119\&lng=en. https:// doi.org/10.5935/0103-104.20140006.

11. Mascarenhas NB, Pereira Á, Da Silva RS, Da Silva MG.
Sistematização da Assistência de Enfermagem ao portador de Diabetes Mellitus e Doença Renal Crônica. Rev. bras. enferm. [Internet]. 2011 Fev [citado 2020 Abr 27] ; 64( 1 ): 203-208. Disponível em: http://www.scielo. br/scielo.php?script=sci_arttext\&pid=S0034-71672011000100031\&lng=en

12. Lata AGB, Albuquerque JG, Carvalho LASBP, Lira ALBC. Diagnósticos de enfermagem em adultos em tratamento de hemodiálise. Acta paul. enferm. [Internet]. 2008 [citado 2020 Abr 27] ; 21( spe ): 160-163. Available from: http://www.scielo.br/scielo.php?script=sci arttext\&pid=S0103-21002008000500004\&lng=en. https://doi.org/10.1590/ S0103-21002008000500004

13. Seymen, P. et al. Evaluation of visual evoked potentials in chronic renal failure patients with diferente treatment modalities. [Internet]. 2010 [citado 2020 Abr 27]; 23(6):705-10. Disponível em: https://pubmed.ncbi. nlm.nih.gov/20301086/.

14. Kirsztajn GM, Bastos MG, Andriolo A. Dia Mundial da Borda 2011 Proteína e creatina sérica: testes essenciais para diagnóstico de doença renal crônica. J. Bras. Patol. Med. Lab. [Internet]. Abr 2011 [citado 2020 abr 27]; 47 (2): 100-103. Disponível em: http://www.scielo.br/scielo.php?script=sci arttext\&pid=S1676-24442011000200002\&lng=en. https://doi.org/10.1590/ S1676-24442011000200002

15. Rosa KR, Loures MC. Qualidade de vida de idosos em hemodiálise: Enfermagem e o Lúdico. [Internet]. dez. 2013 [citado em 2020 em 27 de abril]; 40, n. 4, p.419-446. Disponível em: file://C:/Users/Ultimater/ Downloads/3050-9056-1-PB\%20(1).pdf

16. Riella, MC. Princípios de Nefrologia e Distúrbios hidroeletrolíticos. $5^{\mathrm{a}}$ ed. Rio de Janeiro: Guanabara Koogan, 2010.

17. Maniva, S. J. C. F.; Freitas, C. H. A. O paciente em hemodiálise: autocuidado com a fístula arteriovenosa. Revista da Rede de Enfermagem do Nordeste-Rev Rene, [Internet] 2010; [acesso em Abr 2020] v. 11, n. 1, 2012. Disponível em: http://www.revistarene.ufc.br/revista/index.php/revista/ article/view/358.

18. Nascimento CD, Marques IR. Revisão da literatura. Rev. bras. enferm. [Internet]. $2005 \mathrm{Dez}$ [citado $2020 \mathrm{Abr}$ 27] ; 58( 6 ): 719 722. Disponível em: http://www.scielo.br/scielo.php?script=sci arttext\&pid=S0034-71672005000600017\&lng=en. https://doi.org/10.1590/ S0034-71672005000600017.

19. Daugirdas, J. T.; Blake, P.G.; Ing, T. S. Handbook of dialysis Lippincott Williams \& Wilkins, 2012. ISBN 978-1-4511-4429-

20. Ludlow, V. Buttonhole cannulation in hemodialysis: Improved outcomes and increased expense - Is it worth it? Journal ACITN, [Internet] 2010; [acesso em Abr 2020]. v. 20, n. 1, p. 29, 2010. Disponível em: http:/ www.biomedsearch.com/article/Buttonhole-cannulation-inhemodialysisimproved/223216275.html.

21. Nunes L, Menezes O. O bem-estar, a qualidade de vida e a saúde dos idosos. Lisboa. Editorial Caminho, 2014

22. Silveira CB, Pantoja IKOR, Silva ARM, Azevedo RN, Sá NB, Turiel MGP et al . Qualidade de vida de pacientes em hemodiálise em um hospital público de Belém - Pará. J. Bras. Nefrol. [Internet]. 2010 Mar [citado 2020 Abr 27]; 32( 1 ): 39-44. Disponível em: http://www.scielo.br/scielo. php?script=sci_arttext\&pid=S0101-28002010000100008\&lng=en. https:// doi.org/10.1590/S0101-28002010000100008.

23. Simeão, S. F. A. P. et al. Qualidade de vida de estudantes do ensino médio. [Internet]. 2010 [citado 2020 Abr 27]; R. bras. Qual. Vida, Ponta Grossa, v. 8, n. 3, p. 218-228. Disponível em: https://periodicos.utfpr.edu.br/ rbqv/article/download/4090/3225.

24. Rodrigues J, Mandelbrot DA, Hanto DW, Johnson SR, Karp SJ Pavlakis M. A cross-sectional study of fatigue and sleep quality before and after kidney transplantation. Clin Transplant. 2011 Jan/Fev; v. 25 n. 1 p.1321

25. Carvalho MFD, Moreira MRCN, Nunes CM. Estágios do pesar nos discursos de jovens em tratamento renal substitutivo. Rev enferm UERJ [Internet]. 2012 [cited 2020 Abr 27];20(2):203-8. Available from: http:// www.epublicacoes.uerj.br/index.php/enfermagemue rj/article/view/4052 8 . Barreto MDS, Marcon

26. Medeiros R.H., Pinent C.E. DA C., Meyer F. Aptidão física de indivíduo com doença renal crônica. Jornal Brasileiro de Nefrologia; v. 24 n. 2 p.81-7. [Internet]. jun. 2002 [citado 2020 Abr 27]; 24(2):81-87,. Disponível 
em: http://bases.bireme.br/cgi-bin/wxislind.exe/iah/online/?IsisScript=iah/ iah.xis\&src=google\&base=LILACS\&lang $=$ p\&nextAction=lnk\&exprSearch $=315349$ \&indexSearch $=$ ID

27. Souza FTZ, Oliveira JHA. Sintomas depressivos e ansiosos no paciente renal crônico em tratamento conservador. Rev. Psicol. Saúde [Internet]. 2017 Dez [citado 2020 Abr 27] ; 9( 3 ): 17-31. Disponível em: http://pepsic.bvsalud.org/scielo.php?script=sci arttext\&pid=S2177093X2017000300002\&lng=pt. http://dx.doi.org/10.20435/pssa.v9i3.429

28. Gesualdo GD, Menezes ALC, Rusa SG, Napoleão AA, Figueiredo RM, Melhado VR, et al . Fatores associados à qualidade de vida de pacientes em hemodiálise. Texto contexto - enferm. [Internet]. 2017 [cited 2020 Abr 27] ; 26( 2 ): e05600015. Disponível em: http://www.scielo.br/scielo. php?script=sci_arttext\&pid=S0104-07072017000200338\&lng=en. Epub July 10, 2017. https://doi.org/10.1590/0104-07072017005600015

29. Oliveira APB, Schmidt DB, Amatneeks TM, Santos JC, Cavallet LHR, Michel RB. Qualidade de vida de pacientes em hemodiálise e sua relação com mortalidade, hospitalizações e má adesão ao tratamento. J. Bras. Nefrol. [Internet]. 2016 Dez [citado 2020 Abr 27]; 38(4):411-420. Disponível em: $\quad$ http://www.scielo.br/scielo.php?script=sci arttext\&pid=S010128002016000400411\&lng=en. https://doi.org/10.5935/01012800.20160066

30. Santos BP, Oliveira VA, Soares MC, Schwartz E. Doença Renal Crônica: relação dos pacientes com a hemodiálise. ABCS Health Sci. [Internet]. 2017 Abr. 26 [citado 2020 Abr. 27]; 42(1). Disponível em: https:// www.portalnepas.org.br/abcshs/article/view/943 